\title{
Lifi technology in Future Benefits in Several sectors
}

\author{
Md. Saiful Bari
}

Department of Brac Business School, Brac University, Bangladesh.

\begin{tabular}{llc}
\hline \multicolumn{3}{l}{ Article Information } \\
Received & $:$ & 02 July 2020 \\
Revised & $:$ & 15Augusy 2020 \\
Accepted & $:$ & 18 August2020 \\
Published & $:$ & 24 August2020
\end{tabular}

Corresponding Author:

\section{Md. Saiful Bari}

Email:

md.saiful.bari@g.bracu.ac.bd

\begin{abstract}
Whether you are the usage of wireless net in an espresso shop, stealing it from the man subsequent door, or competing for bandwidth at a conference, you have probable gotten pissed off at the gradual speeds you face when extra than one system is tapped into the network. But radio waves are clearly one section of the spectrum that can raise our data. What if we can also wish to use exclusive waves to surf the internet? One German physicist. Harald Haas, has come up with a reply he calls "Data Through Illumination"- taking the fiber out of fiber optics with the resource of potential of sending records thru an LED moderate bulb that varies in intensity faster than the human eye can follow. And safety would be a snap-if you can't see the light, you can't get entry to the data. Li-Fi is a VLC, considered slight communication, technological perception developed by way of using a crew of scientists collectively with $\mathrm{Dr}$ Gordon Povey, Prof. Harald Haas and Dr Mostafa Afgani at the University of Edinburgh. The time size Li-Fi used to be coined via skill of the use of Prof. Haas when he amazed humans by means of potential of means of streaming high- definition video from a modern-day LED lamp, at TED Global in July 2011. Unseen with the resource of practicable of the human eye, this variant is used to elevate high-speed data," says Dr Povey, Product Manager of the University of Edinburgh's Li-Fi Program 'D-Light Project'.
\end{abstract}

Keywords: Li-Fi (Light Fidelity), RF (Radio Frequency), LED (Light Emitting Diode)

Copyright (C) 2020: Md. Saiful Bari. This is an open access distribution, and reproduction in any medium, provided Access article distributed under the Creative Commons Attribution License the original work is properly cited License, which permits unrestricted use.

Citation: Md. Saiful Bari. "Lifi technology in future benefits in several sectors, "Journal of Science, Computing and Engineering Research, 1(3), 67-72, July-August 2020.

\section{INTRODUCTION}

$\mathrm{Li}-\mathrm{Fi}$ (also regarded as slight fidelity) is a science for the wi-fi transmission of data between gadgets Li-Fi makes use of moderate as a medium for transmission of data. LED lamps are the supply used for encoding information by means of switching the light on and off for the length of normal intervals to create a binary code. This is transmitted to the precise supply up the vicinity the receiver decides it. $\mathrm{Li}-\mathrm{Fi}$ has a large achievable in information transmission when you reflect on consideration on that it makes use of easy LED bulbs to transmit data.

According to [2], $\mathrm{LiFi}$ and $\mathrm{WiFi}$ has the difference associated to the congestion, density, security, safety, and speed. The greater WiFi enabled device is exist, the congestion may additionally moreover occur. In the science of WiFi we can't add extra routers if the man or woman is increased, at the same time as we can add the moderate in LiFi. Efficiency and safety of the net are the dominating troubles proper now. The common performance of LiFi is claimed that greater better than the regular overall performance of $\mathrm{WiFi}$. The fee tempo of $\mathrm{LiFi}$ is one thousand instances quicker than WiFi. For safety of the internet, LiFi is large tightly closed than the WiFi based totally on the unfold of the signal.

LiFi has a light characteristic that average cannot go by means of the wall. It is one-of-a-kind from the signal of
WiFi can go via anywhere. Based on these two technologies, in an easy conclusion is LiFi has larger tightly closed communication then again than the WiFi. The vulnerability exists if there are leakage in the wall whilst having an indoor communication. Security threat that would possibly additionally moreover exist is an intruder can spoof the information the use of the leakage wall.

This paper provides a clarification of the newly developed science that is LiFi. The paper will discuss about the architecture, modulation, performance, and the challenges. The shape of this paper is as follows: in the remaining section of this segment we will speak about the LiFi idea based totally on VLC. In location four we similarly analyze the average overall performance of $\mathrm{LiFi}$ based on the charge tempo and safety evaluating with the WiFi. In place 5 we speak about the challenge in $\mathrm{LiFi}$ technology, it consists of a future lookup based on the protection issue. All the stop results of According to, $\mathrm{LiFi}$ and $\mathrm{WiFi}$ has the big difference associated to the congestion, density, security, safety and speed. The extended WiFi enabled gadget is exist, the congestion would possibly additionally occur. Efficiency and safety of the net are the dominating troubles gorgeous now. The average performance of $\mathrm{LiFi}$ is claimed that large better than the frequent standard performance of $\mathrm{WiFi}$. The charge pace of $\mathrm{LiFi}$ is one thousand situations quicker than WiFi. The structure of this paper is as follows: in the last area of this 
vicinity we will talk about the LiFi thinking surprisingly based totally on VLC. In section three we discuss about the structure of LiFi. In neighborhood 4 we in addition analyze the typical performance of LiFi based on the charge speed and safety evaluating with the WiFi. In part 5 we discuss about the challenge in LiFi technology, it consists of a future lookup based certainly on the security issue. All the result of According to, $\mathrm{LiFi}$ and $\mathrm{WiFi}$ has the difference associated to the congestion, density, security, safety, and speed. The higher WiFi enabled gadget is exist, the congestion may also moreover occur. In the science of WiFi we can't add large routers if the man or woman is increased, even as we can add the reasonable in LiFi. Efficiency and protection of the net are the dominating troubles proper now. The average overall performance of $\mathrm{LiFi}$ is claimed that greater than the regular performance of $\mathrm{WiFi}$. The price tempo of $\mathrm{LiFi}$ is one thousand times faster than WiFi.

For safety of the internet, LiFi is greater tightly closed than the WiFi primarily based completely on the unfold of the signal. LiFi has a mild attribute that slight can't go via the wall. It is one-of-kind from the signal of WiFi can go via anywhere. Based on these two technologies, in a reachable conclusion is $\mathrm{LiFi}$ has more invulnerable dialog as an alternative than the WiFi. The vulnerability exists if there are leakage in the wall even as having an indoor communication. Security hazard that can also exist is an intruder can spoof the data the use of the leakage wall. This paper offers an explanation of the newly developed technological expertise that is $\mathrm{LiFi}$.

The paper will talk about the architecture, modulation, performance, and the challenges. The shape of this paper is as follows: in the closing segment of this phase we will talk about the LiFi notion basically based totally on VLC. In part three we discuss about the structure of LiFi. In phase 4 we in a similar way analyze the performance of LiFi principally based totally on the rate tempo and protection evaluating with the WiFi. In phase 5 we discuss about the undertaking in LiFi technology, it consists of a future lookup based absolutely on the protection issue. All the give up quit end result of this paper is summarized in phase 6 . The end gives up end result of this paper can be used as a reference and perception when creating a LiFi technology.

According to determine 1, LiFi science consist of LED Lamp as the media transmission and picture detector as a receiver of transmitted data. Lamp driver is wished to make LED working properly. While amplification and processing are responsible to manipulate the sign that comes from the image detector. Basic wondering for working precept in LiFi Technology are pointing into: Transceiver and Light as a media transmission. Figure 2 is a predominant thinking block sketch for LiFi. This essential thinking suggests as a duplex communication. The costs of $\mathrm{LiFi}$ is $14 \mathrm{Gbps}$ the utilization of three off- the- shelf laser diodes (red, green, and blue) and predict the fee until 100Gbps when the whole seen spectrum is used.
According to [3] $\mathrm{LiFi}$ and VLC used a comparable medium as an information dialog that is light. The distinction between LiFi and VLC is VLC has a unidirectional, point-to-point mild dialog at low statistics rates. While the $\mathrm{LiFi}$ science is utterly networked, bidirectional, and high-speed WiFi communication. Others stated LiFi is the incorporation of WiFi and VLC [2].

Due to the creating demand for Wi-Fi records communication, the available radio spectrum beneath 10 $\mathrm{GHz}$ (cmwave communication) has develop to be insufficient. The wi-fi communication business enterprise has answered to this mission by means of way of way of considering the radio spectrum above $10 \mathrm{GHz}$ (mm-wave communication). However, the large frequencies, f, suggest that the direction loss, L, increases in accordance to the Frias free place equation ( $\mathrm{L} \quad \mathrm{F} 2$ ). In addition, blockages and shadowing in terrestrial dialog are extra tough to overcome at large frequencies. As a consequence, constructions ought to be designed to beautify the chance of line of-sight (LoS), generally via the usage of the use of beam forming methods and by means of way of way of the usage of very small cells (about $50 \mathrm{~m}$ in radius). The prefer for small cells is now no longer a situation from a gadget functionality perspective. This is due to the truth reducing cell sizes has barring doubt been the essential contributor for top of the line gadget frequent performance in current day cellular communications. This means, opposite to the everyday understanding, the use of expanded frequencies for terrestrial verbal alternate has given up a realistic option. However, one draw returned is that the venture for providing an assisting infrastructure for ever smaller cells will grow to be significant. One such instance is the provision of a sophisticated backhaul infrastructure. Lightfidelity ( $\mathrm{LiFi}$ ) [1], [2] is a continuation of the fashion to go to larger frequencies in the electromagnetic spectrum. Specifically, LiFi ought to be classified as.

\section{SCOPE OF THE STUDY}

In this paper two we strive lifi science in future. The two Li-Fi two science two can two be two used two for relatively a range purposes, two hits things two the data two transmission two via LEDs hence all the shows which illuminate mild can be served as a platform for data communication. The screen of the telephone phone, television, bulbs can act as a supply of light. On the special hand, the receiving platform, two the image two detector can two be modified with the resource of capability of two a digital camera in mobile phone smartphone for two scanning and retrieving data. two Its different two functions are two Li-fi two for desktops, smartcard two LiFi, Li-fi for schools, hospitals, Li-fi in cities, two sensible two guides, museums, hotels, fairgrounds, occasions indoor and LBS(Locationbased two Services), get entry to manipulate and identification crisis, malls, airport and unstable environments like thermal two power plants.[7]It moreover has the benefit of being beneficial in electromagnetic touchy areas such two as in two plane cabins, two hospitals and two 
nuclear two energy plants two barring inflicting electromagnetic interference IV. REAL TIME USAGE OF LI-FI two Fig. 1 shows the real time use of $\mathrm{LiFi}$ Technology. Li-Fi can be used at the vicinity of Wi-Fi for net connection two to all two devices. It is moreover very two beneficial for dialog between two units for records switch and one of-a-kind two variety two of connections. It's two offers the very hastily speed for web get entry to two and streaming cause and moreover very shortly and impenetrable records switch between the devices. So, the LiFi Technology two is very advocated for ordinary use like at the location of Wi-Fi and Other wi-fi two applied sciences two for statistics two transmission two or net access.

\section{LIMITATION OF STUDY}

The Limitations of LiFi Study are as follows:

1. The learn about is restricted to LiFi in future only.

2. The locate out about is notably based truly upon the $\mathrm{LiFi}$ technological perception in future benefits and how to use $\mathrm{LiFi}$

3. The data accrued for the seem to be up is surely on primary data given with the aid of using the respondents. There is danger for personal bias.

\section{OBJECTIVES}

The excessive intention of the venture is to create a software that transmits information be it text, audio or video the usage of Li-Fi technological know-how for coping with the restricted bandwidth hassle we face in RF (Radio frequency) signals. For better, efficient, impenetrable and a quicker connection Li-Fi is used. One of the blessings of the usage of $\mathrm{Li}-\mathrm{Fi}$ over $\mathrm{Wi}-\mathrm{Fi}$ is that it avoids radiation produced with the useful resource of $\mathrm{Wi}-\mathrm{Fi}$. The motive of our challenge is to change information with quicker tempo which is now not common to achieve through Wi-Fi and see whether or now not or now not transmission is viable via capability of tremendously a quantity medium or not. Li-Fi can be thought as a desire for Wi-Fi which makes use of mild as a medium to transmit data. We aimed at the transmission of statistics through Li-Fi and all the feasible two components of transmitting facts by means of skill of way of slight with the aid of capacity of a variety medium which will furnish us a massive thinking of where and how we can use Li-Fi for transmission of data.
1. LiFi Technology
is primarily based on
all a differ of sorts of

$$
\text { light. }
$$

2. In the world's mild is present everywhere, and there are many bulbs, the definitely issue is to alternate with the aid of LED's for the information transmission.

3. Light has ten thousand instances wider bandwidth than radio waves that proves greater capacity

4. Light cannot penetrate the walls, which affords the security.

5. LiFi is very extra price extremely good for statistics transmission purpose.

6. WiFi can't be use beneath water. But, LiFi can be, moderate can be used underneath the water.
7. By the use of LiFi technology, every avenue average someplace would be free files hotspot.

8. LiFi in no way damage dwelling aspect whereas radio waves have an effect on the human body, birds and plants.

9. LiFi technological perception is recommended in nuclear sector, airlines, electricity plants, and etc.

10. LiFi science gives smart way to manipulate traffics. It is relevant to GPS utilization

\begin{tabular}{llllll} 
Valid & Age & Frequency & Percent & $\begin{array}{l}\text { Valid } \\
\text { Percent }\end{array}$ & $\begin{array}{l}\text { Cumulative } \\
\text { percent }\end{array}$ \\
\hline YES & $10-18$ & 15 & $10 \%$ & $10 \%$ & $10 \%$ \\
YES & $19-26$ & 50 & $33 \%$ & $33 \%$ & $43 \%$ \\
YES & $27-34$ & 40 & $27 \%$ & $27 \%$ & $70 \%$ \\
YES & $35-42$ & 35 & $23 \%$ & $23 \%$ & $93 \%$ \\
YES & $42-50$ & 10 & $7 \%$ & $7 \%$ & $100 \%$ \\
Total & & 150 & $100 \%$ & $100 \%$ &
\end{tabular}

\section{RESEARCH METHODOLOGY}

This file has been developed more often than not based on most vital and secondary statistics related to lifi science in future. Secondary information's are assembled from Books, Journals, Research paper, online archives etc. Primary information is gathered via perception and interview method. Followings are the quintessential methodologies that has been used to put at the same time this report, this archive has been accumulated through a selfsurvey questioner. Research method: It will be a mixture of exploratory and casual study.

1. Population: Here populace will be every physique who does use LiFi technology.

2. Sample: in this research, alleviation and random sampling technique will be used.

3. Sample size: Here sample dimension will be a hundred and twenty people.

4. Statistical software: SPSS will be used in this research.

5. Data contrast strategy: Statistical gadget is used in this research. Data collection: The self-survey questionnaire is used to accumulate the data. Total Population: We interview one hundred fifty humans survey the human beings use LiFi.

\section{A. Data Analysis and Interpretation}

1. Age: Any Age of Person

\section{What is your Age?}

From the above table, we can see that $10 \%$ of the respondents are 1018-year age group $33 \%$ of the respondents 19-26-year age group $27 \%$ of the respondents 27-34-year age group $23 \%$ of the respondents 3542-year age 
group $7 \%$ and the rest of the respondents are 42-50-year age group. As we can see most of the 19-42 age are use LiFi technology because $\mathrm{LiFi}$ technology are easier to use for future.

\section{Occupation: Any Occupation}

\section{What is your Occupation?}

\begin{tabular}{llllll} 
Valid & $\begin{array}{l}\text { Occupant- } \\
\text { ton }\end{array}$ & $\begin{array}{l}\text { Fre } \\
\text { que } \\
\text { ncy }\end{array}$ & Percent & $\begin{array}{l}\text { Valid } \\
\text { Perce } \\
\text { nt }\end{array}$ & $\begin{array}{l}\text { Cumulative } \\
\text { Percent }\end{array}$ \\
\hline YES & Student & 45 & $30 \%$ & $30 \%$ & $30 \%$ \\
YES & Engineer & 40 & $27 \%$ & $27 \%$ & $57 \%$ \\
YES & Job & 30 & $20 \%$ & $20 \%$ & $77 \%$ \\
& Holder & & & & \\
YES & Business & 20 & $13 \%$ & $13 \%$ & $90 \%$ \\
& Holder & & & & \\
YES & Doctor & 10 & $7 \%$ & $7 \%$ & $97 \%$ \\
YES & Others & 5 & $3 \%$ & $3 \%$ & $100 \%$ \\
Total & & 150 & & &
\end{tabular}

From the above table, we can see that $30 \%$ of the respondents are students, $27 \%$ of the respondents are engineers, $20 \%$ of the respondents are job holders $13 \%$ of the business men holders $7 \%$ of the doctors and the rest $3 \%$ of the respondents are others. As we can see students and engineers are most demanded LiFi technology.

3. Gender: Male and Female

What is your Gender?

Valid Gender Frequency Percent Valid CumulaPercent

tive

Percent

$\begin{array}{llllll}\text { YES } & \text { Male } & 90 & 60 \% & 60 \% & 60 \% \\ \text { YES } & \text { Female } & 60 & 40 \% & 40 \% & 100 \% \\ \text { YES } & \text { Total } & 150 & 100 \% & 100 \% & \end{array}$

From the above table, we can see that $60 \%$ of the respondents are male and $40 \%$ of the respondents are female. As we see male are so interested lifi technology. Female is moderate.

Dense Urban Environments (Interference-free Wireless Communication) Dense metropolis environments cowl large region with synthetic lighting. The area with lighting infrastructure can provide excessive data price get proper of entry to for customers as the clients pass via that environment. For example, in a hotel hall or reception hall a variety of users can acquire high data price at any point. Moreover, excessive speed Wi-Fi verbal change would be accessible in every room thinking about the light waves cannot propagate thru walls. This effects in interference-free wireless communication. Other employments of Li-Fi innovation in backyard urban territory is that $\mathrm{Li}-\mathrm{Fi}$ can empower road lights that would give a gadget of get proper of entry to focuses for web. In telephone correspondence, the separation between radio base stations is less. In this way, instead than conveying new radio base stations in our metropolis areas, street lights ought to furnish both, light amid night and data correspondence at International Journal of Information Sciences and Techniques rapid every and each and every minute of every day. In this way, charge of arrangement and group of new radio base station can be spared. Shockingly, alternatively when the lights are off as saw by the eye, immoderate data correspondence prices are though conceivable. EMI Sensitive Environments Use of Li$\mathrm{Fi}$ in aircraft, allow passenger to take the advantage of high records rate connectivity at all-time barring producing electromagnetic interference (EMI) interference (EMI) with sensitive radio equipment on the flight deck. The reduction in cabling requirement additionally capability a lighter aircraft.

\section{RECENT TECHNOLOGIES}

\section{A. Augmented Reality}

Exhibitions in museums and galleries are illuminated with artificial lighting. Li-Fi technological know-how enables lighting can supply localized information. So that a visitor $\mathrm{s}$ digicam or cellular telephone can be used to download in a similar way statistic related to the object being seen from the light. two Localized

\section{B. Advertising}

By merging avenue lights and lights display workable of a save as a Li-Fi broadcast channel, it is viable to transmit advertising and advertising facts like magnificent presents and coupons. So, this gives probability to emerge novel retail enterprise model. Product information, catalogue information, cut charge coupons and advertising videos ought to all be provided to shoppers. Two Safety Environments the use of electrical tools like telephone telephones in explosion hazard environments is generally restricted. With the use of $\mathrm{Li}-\mathrm{Fi}$ science to omit records will simplify the configuration of data networks and can enable new buildings to embellish safety in these environments.

\section{Intelligent Transportation Systems}

Now-a-days headlights and tail lights of vehicle are step by step being changed with LED versions. This offers the possibility of car-to-car dialog the use of Li-Fi, permitting enchantment of anti-collision systems and change of data about driving stipulations between vehicles. Traffic lights can additionally be used that already use LED lighting, so it offers the opportunity to manage traffic systems. This would additionally permit automobile buildings to down load 
statistics on hand on the network related to optimum routes to take, and change the community related to stipulations recently experienced thru man or lady vehicles.

\section{Connectivity}

Our houses as of now have lights fixtures extensively introduced. The utilization of LiFi empowered lighting will alternate the purposes that can be conceived, now not simply the interconnection of gadgets, for example, TVs, PCs and $\mathrm{Hi}-\mathrm{Fi}$, moreover interfacing regular regional apparatuses, for example, ice chests, clothes washers, microwaves and vacuums. The "web of everything". For safety of the internet, $\mathrm{LiFi}$ is extra tightly closed than the $\mathrm{WiFi}$ exceptionally mainly based on the unfold of the signal. LiFi has a mild characteristic that moderate cannot go with the useful resource of the wall. It is extremely good from the sign of WiFi can go through the use of anywhere. Based on these two technologies, in a handy conclusion is LiFi has extra tightly closed communication as a choice than the WiFi. The vulnerability exists if there are leakage in the wall even as having an indoor communication. Security hazard that can also moreover exist is an intruder can spoof the information the utilization of the leakage wall. This paper affords an explanation of the newly developed science that is LiFi. The paper will discuss about the architecture, modulation, performance, and the challenges. The shape of this paper is as follows: in the closing segment of this location we will discuss about the LiFi thinking particularly based absolutely on VLC. In section three we discuss about the shape of LiFi. In vicinity four we in a comparable way analyze the trendy overall performance of LiFi based on the cost pace and protection evaluating with the $\mathrm{WiFi}$. In place 5 we speak about the challenge in LiFi technology, it consists of a future seem to be up in particular primarily based on the safety issue. All the end result of According to, LiFi and WiFi has the big distinction associated to the congestion, density, security, safety, and speed. The larger WiFi enabled device is exist, the congestion might also occur. In the technological expertise of WiFi we can't add accelerated routers if the client is increased, even as we can add the moderate in LiFi. Efficiency and safety of the internet are the dominating problems splendid now. The overall performance of $\mathrm{LiFi}$ is claimed that better than the typical performance of $\mathrm{WiFi}$. The fee speed of $\mathrm{LiFi}$ is a thousand instances quicker than WiFi.

For protection of the internet, LiFi is more tightly closed than the WiFi mainly primarily based on the unfold of the signal. LiFi has a mild characteristic that mild can't go by using way of the wall. It is great from the sign of WiFi can go thru anywhere. Based on these two technologies, in an undemanding conclusion is $\mathrm{LiFi}$ has more impenetrable communication however than the $\mathrm{WiFi}$. The vulnerability exists if there are leakage in the wall even as having an indoor communication. Security hazard that would possibly also exist is an intruder can spoof the files the use of the leakage wall. This paper presents an explanation of the newly developed technological expertise that is LiFi. The paper will discuss about the architecture, modulation, performance, and the challenges. The structure of this paper is as follows: in the closing part of this location we will speak about the LiFi notion chiefly primarily based sincerely on VLC. In section three we talk about the shape of LiFi. In vicinity four we in a similar fashion analyze the usual general performance of LiFi based on the fee tempo and safety evaluating with the WiFi. In part 5 we speak about the challenge in LiFi technology, it consists of a future search for specifically based totally on the safety issue.

\section{CONCLUSION}

All the end result of According to, $\mathrm{LiFi}$ and $\mathrm{WiFi}$ has the distinction associated to the congestion, density, security, safety, and speed. The greater WiFi enabled laptop is exist, the congestion would per chance moreover occur. In the science of WiFi we can't add extra routers if the customer is increased, whilst we can add the moderate in $\mathrm{LiFi}$. Efficiency and safety of the web are the dominating troubles suited now. The average overall performance of $\mathrm{LiFi}$ is claimed that larger better than the performance of WiFi. The price tempo of $\mathrm{LiFi}$ is one thousand times faster than $\mathrm{WiFi}$. For security of the internet, LiFi is expanded tightly closed than the WiFi in unique in particular based on the unfold of the signal. LiFi has a slight characteristic that slight can now not go through the wall. It is unique from the sign of $\mathrm{WiFi}$ can go via anywhere. Based on those two technologies, in a convenient conclusion is $\mathrm{LiFi}$ has larger invulnerable communication then again than the $\mathrm{WiFi}$. The vulnerability exists if there are leakage in the wall at the same time as having an indoor communication. Security threat that might moreover exist is an intruder can spoof the records the use of the leakage wall. This paper affords a rationalization of the newly developed science that is LiFi. The paper will discuss about the architecture, modulation, performance, and the challenges.

\section{REFERENCES}

[1]. Mahendran, R., 2016, May. Integrated Li-Fi (Light Fidelity) for wise dialog by way of the usage of illumination. In Advanced Communication Control and Computing Technologies (ICACCCT), 2016 International Conference on (pp. 53-56). IEEE. He the methodology of sending photo from one device to another the use of Li-Fi technology.

[2]. H. Haas and N. Serafimovski, "Li-Fi unlocking unprecedented wi-fi pathways for our digital future, IEEE ComSoc Technology News, Dec. 2016.

[3]. In November 2016, IEEE has fashioned a Topic Interest Group (TIG) to begin matters to do of LiFi standardization within the IEEE wi-fi LAN Standard P802.11. The group is lead by way of way of pureLiFi and has many supporting institutions (a non exhaustive list consists of Cisco, LG Electronics, Lucibel, Schneider, Nokia, Boeing, Hewlett Packard, and Fraunhofer).

[4]. T. Komine and M. Nakagawa, "Fundamental analysis for seen moderate dialog buildings the use of LED Lights, "IEEE Trans. Consumer Electronics, vol. 59, no.1, Feb, 2004.

[5]. P. Amirshahi, M. Kavehrad, "Broadband Access over Medium and Low Voltage Power traces and use of White 
Light Emitting Diodes for Indoor Communications, ” IEEE Consumer Communications \& Networking Conference, Las Vegas, Nevada, January 2006. Optical Wireless Communications Using White LED Lighting".

[6]. http://www.lifi-lab.com/lifi/lifi-enhanced-mode-futurewireless-communicationreview.html.

[7]. http://www.lifi-centre.com/about-li-fi/applications/.

[8]. warudkar, r. S., \&amp; malani, s. S. Li-fi: a new strategy in wi-fi communication. mihir chauhan and aditya kulai (2015), li-fi let there be light, worldwide journal of engineering traits and technology (ijett) quantity 28.

[9]. Haas H. Wireless records from every and each slight bulb. TED Website TEDGlobal, Edinburgh, Scotland. 2011.

[10]. Haas H, Chen C. What is LiFi? J Light Technol. 2015;34:1533-44.Website: http:/www.lificonsortium.org.

[11].Edinburgh. PR. "pureVLC Demonstrates Li-Fi Streaming alongwith Research Supporting World's Fastest Li-Fi Speeds up to 6Gbit/s." 2013.

[12].Li-Fi Internet reply from the Russian agency attracting foreignclients, Russia, and India Report. 2014.

[13].Vega A "Li-fi record statistics transmission of $10 \mathrm{Gbps}$ set the use of LED lights." Engineering and Technology Magazine [Internet]. 2014;

[14].Available from: https://eandt.theiet.org/content/articles/ 2014/07/li-fi-record-data-transmission-of-10gbps-setusingled-lights/ Accessed on 22 June 2018.

[15].Povey G. Top 10 Li-Fi myths. Visible Light Commun. 2012.Available from: http://visiblelightcomm.com/top-10-lifimyths/Accessed on 22 June 2018.

[16]. Fields RD. Mind administration by way of the skill of a phone phone. Sci Am. 2014. Available from: https://www.scientificamerican.com/article/mind-control-bycell/ Accessed on 22 June 2018.

[17]. Cunnington D, Junge MF. Insomnia: prevalence, consequences, and terrific treatment. Med J Aust. 2013;199(8):36-40.

[18].Pyrpasopoulou A, Kotoula V, Cheva A, Hytiroglou P, Nikolakaki E, Magras IN, et al. Bone morphogenetic protein expression in newborn rat kidneys after prenatal publicity to radiofrequency radiation. Bioelectromagnetics. 2004;25(3):216-27.

[19].Lv B, Chen Z, Wu T, Shao Q, Yan D, Ma L, et al. The alteration of spontaneous low-frequency oscillations precipitated with the aid of acuteelectromagnetic discipline exposure. Clin Neurophysiol. 2013;125(2):277-86.

[20]. Shahin S, Singh VP, Shukla RK, Dhawan A, Gangwar RK, Singh SP. $2.45 \mathrm{GHz}$ microwave irradiation-induced oxidative stress affects implantation or being pregnant in mice, mus musculus. Appl Biochem Biotechnol. 2013;169(5):1727-51.

[21]. Havas M, Marrongelle J. Replication of coronary heart price variability provocation study about with $2.45 \mathrm{GHz}$ cordless smartphone confirms original findings. Electromagn Biol Med. 2013;32(2):253-66.

[22].Rani J, Chauhan P, Tripathi R. Li-Fi (Light Fidelity)-the futuretechnology in wi-fi communication. Int J Appl Eng Res.2012;7:1517-20.

[23]. Jaiswal NS, Chopade PS. Technology- moderate bulb to get proper of entry to the Internet! consider of $\mathrm{Li}-\mathrm{Fi}$ Technology: new Future. Int J Sci Eng Res. 2013;4:36-40.

[24]. Gupta SU. Research on Li-Fi science \&amp; comparison of $\mathrm{Li}-\mathrm{Fi} / \mathrm{Wi}-\mathrm{Fi}$. International Journal of Advanced Research in Computer Science and Software Engineering. 2015;5(6):429-33.
[25]. Chatterjee S. Scope and challenges in mild fidelity (LiFi) technology in wi-fi records communication. Int $\mathrm{J}$ Innov ResAdv Eng. 2015;2:1-9.

[26]. Ayyash M, Elgala H, Khreishah A, Jungnickel V, Little T, Shao $\mathrm{S}$, et al. Coexistence of $\mathrm{WiFi}$ and $\mathrm{LiFi}$ closer to $5 \mathrm{G}$ : concepts, opportunities, and challenges. IEEE Commun Mag. 2016;54:64-71.

[27].Mishra P, Poddar J, Priya S, A overview on LiFi: the inexperienced WiFi. Int Res J Eng Technol. 2016;3:99-103.

[28]. Shao S, Khreishah A, Rahaim MB, Elgala H, Ayyash M, LittleTD, et al. An indoor hybrid WiFi-VLC Internet receives entry to the system.Proc - eleventh IEEE Int Conf Mob Ad Hoc Sens Syst MASS 2014.2015;(Vlc):569-74.

[29]. Shao S, Khreishah A, Ayyash M, Michael B, Rahaim HE, Jungnickel V, et al. Design and assessment of a visible-light communication enhanced WiFi system. IEEE/OSA J Opt CommunNetw. 2015;7(10):960-73.

[30].Dimitrov S, Haas H. Principles of LED Light Communications (Towards Networked Li-Fi). Cambridge University Press, UnitedKingdom. 2015.

[31].Saito Y, Kishiyama Y, Benjebbour A, Nakamura T, Li A, Higuchi K Non-orthogonal a couple of getting admission to (NOMA) for telephone future radio access. In: IEEE Vehicular Technology Conference. Dresden, Germany; 2013.

[32].Liang Y, Xiping W, Haas H. On the ordinary overall performance of non-orthogonal multiple get entry to viewed moderate communication. IEEE twenty-sixth Annual International Symposium on Personal, Indoor, and Mobile Radio Communications (PIMRC), Hong Kong, China, 2015. 